\section{Antihypertensive Therapie bei Senioren: Sturzrisiko steigt nicht an}

\author{
Das Sturzrisiko unter einer antihypertensiven Therapie ist angesichts \\ der hohen Zahl älterer Patienten, die diese Medikamente benötigen, \\ von besonderer Bedeutung.
}

ie vorliegende Studie hat das Sturzrisiko älterer Patienten in Abhän-

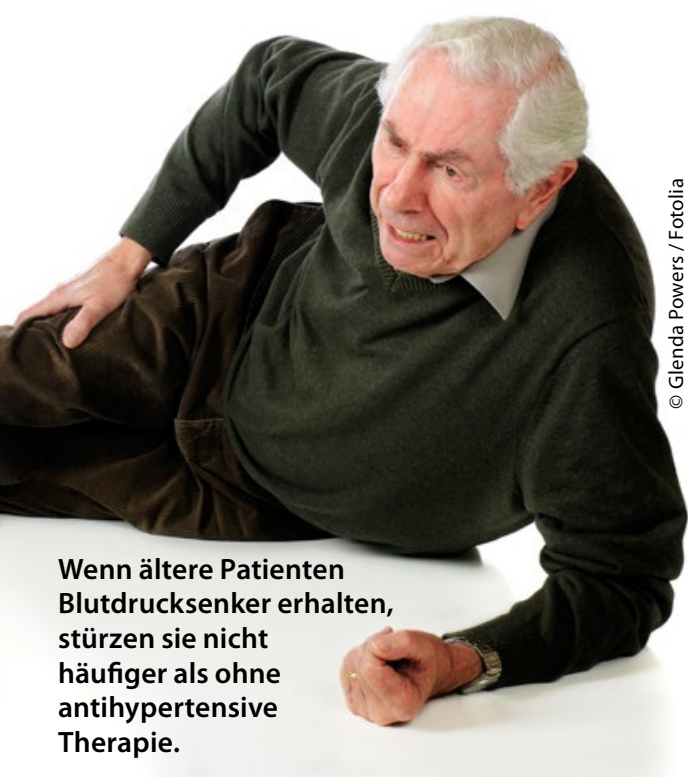

gigkeit vom Blutdruck, der antihypertensiven Medikation sowie den Gebrechlichkeitsindikatoren untersucht. 5.236 Teilnehmer der REGARDS-Studie im Alter von $\geq 65$ Jahren wurden im Mittel 6,4 Jahre beobachtet. Neben dem Blutdruck wurde die AntihypertensivaMedikation sowie die Anzahl der Gebrechlichkeitsindikatoren (niedriger Body-Mass-Index, kognitive Einschränkung, depressive Symptome, Erschöpfung, eingeschränkte Mobilität und Sturzanamnese) und die Häufigkeit von schweren Sturzverletzungen erfasst.

Die Auswertungen zeigen, dass die Inzidenz schwerer Sturzverletzungen weder vom systolischen noch vom diastolischen Blutdruck abhängt. Auch die Anzahl der verwendeten Substanzklassen zur antihypertensiven Behandlung war nicht mit der Häufigkeit schwerer Sturzverletzungen assoziiert. Lediglich die Anzahl von Gebrechlichkeitsindikatoren zeigte einen signifikanten Zusam- menhang zur Inzidenz schwerer Sturzverletzungen.

Bromfield SG et al. Blood Pressure, Antihypertensive Polypharmacy, Frailty, and Risk for Serious Fall Injuries Among Older Treated Adults With Hypertension. Hypertension. 2017;70(2): 259-266.

\section{Kommentar}

Diese Studie zeigt somit, dass die antihypertensive Behandlung älterer Patienten nicht mit einem signifikant gesteigerten Sturzrisiko einhergeht. Die naheliegende Vermutung, dass eine Blutdrucksenkung auch das Risiko von (möglicherweise orthostatisch bedingten) Stürzen steigern könnte, ließ sich an einer großen Probandenzahl mit ausreichend langer Beobachtungsdauer nicht bestätigen. Diese Studie liefert somit ein weiteres Argument für eine konsequente antihypertensive Behandlung älterer Hypertoniker. Der Nutzen der Behandlung dürfte das potenzielle Risiko durch vermehrte Stürze deutlich übersteigen.

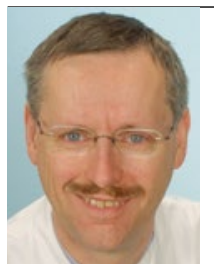

Prof. Dr. med. Walter Zidek Medizinische Klinik IV, Charité Universitätsmedizin Berlin

\section{Schlaganfall-Sekundärprophylaxe mit Ticagrelor: Auf den richtigen Patienten kommt es an}

\author{
In den ersten drei Monaten nach einer transitorischen ischämischen Attacke \\ (TIA) oder einem Hirninfarkt ist die Gefahr eines Rezidivs am höchsten. Mit \\ der Sekundärprophylaxe befasste sich die SOCRATES-Studie.
}

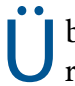
blicherweise wird Acetylsalicylsäure als frühe Sekundärprophylaxe nach einem Hirninfarkt eingesetzt, wenn eine kardiogene Emboliequelle unwahrscheinlich ist. Trotzdem treten auch unter dieser Medikation bei 10$15 \%$ der Patienten Rezidive auf.

In der SOCRATES-Studie wurde deshalb untersucht, ob der Thrombozytenfunktionshemmer Ticagrelor ASS hinsichtlich des kombinierten Endpunkts Tod, Myokardinfarkt und erneuter
}

Schlaganfall überlegen ist. In der Studie wurden 13.199 Patienten mit leichtem Schlaganfall (NIHS $\leq 5)$ oder Hochrisiko-TIA $\left(\mathrm{ABCD}_{2}\right.$-Score 4 oder höher oder symptomatische Stenose intrakranieller oder extrakranieller hirnversorgender Arterien) innerhalb von 24 Stunden randomisiert entweder mit Ticagrelor (Startdosis $180 \mathrm{mg}$, danach $90 \mathrm{mg} 2 \times$ täglich) oder ASS (Startdosis $300 \mathrm{mg}$, danach 100 mg $1 \times$ täglich) über 90 Tage behandelt. Ausschlusskriterien waren eine kardio- embolische Schlaganfallgenese, eine geplante oder durchgeführte revaskularisierende Therapie (i.v. Thrombolyse oder Thrombektomie) oder eine geplante duale Thrombozytenfunktionshemmung. Mithilfe der ASCOD-Einteilung wurden die Patienten hinsichtlich der möglichen Schlaganfallgenese verschiedenen Subtypen zugeordnet.

Das Ergebnis der Studie zeigte zwar einen positiven Trend zugunsten von Ticagrelor, war aber statistisch nicht signifikant. Bei 6,7\% der Patienten in der Ticagrelor-Gruppe und bei 7,5\% der Patienten in der ASS-Gruppe wurde der primäre Endpunkt erreicht ( $\mathrm{p}=0,07)$.

Aufgrund der heterogenen Schlaganfallätiologie in der Studienpopulation wurden in einer aktuellen Subgruppenanalyse Patienten mit einer makroangiopathischen Genese genauer be- 